\title{
Cytogenetic characteristics of 665 patients with myelodysplastic syndrome in China: A single-center report
}

\author{
XUEYING WANG ${ }^{1,2^{*}}$, WEIYI LIU ${ }^{2 *}$, MINGJING WANG ${ }^{2,3}$, TENG FAN $^{2,3}$, YUMENG LI $^{1,2}$, XIAOQING GUO $^{2}$, \\ XIUPENG YANG ${ }^{2}$, HONGZHI WANG $^{2}$, HAIYAN XIAO $^{2}$, SHANSHAN ZHANG $^{2}$, RICHENG QUAN $^{2}$, CHI LIU $^{2}$,

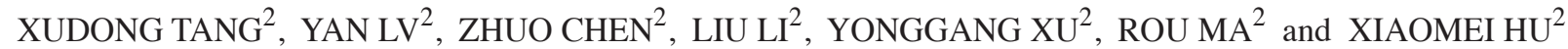 \\ ${ }^{1}$ Graduate School, Beijing University of Chinese Medicine, Beijing 100029; ${ }^{2}$ Department of Hematology, \\ Xiyuan Hospital, China Academy of Chinese Medical Sciences, Beijing $100091 ;{ }^{3}$ Graduate School, \\ China Academy of Chinese Medical Sciences, Beijing 100700, P.R. China
}

Received April 27, 2020; Accepted October 20, 2020

DOI: $10.3892 / \mathrm{ol} .2020 .12387$

\begin{abstract}
The karyotype is highly important for diagnosis and prognosis in myelodysplastic syndrome (MDS). The objective of the present study was to investigate the cytogenetic characteristics of patients with MDS in China. The karyotypes of 665 Chinese patients with MDS were analyzed, and it was identified that 298 cases $(298 / 665,44.8 \%)$ had abnormal karyotypes. Among the 298 patients with abnormal karyotypes, the 75 patients with trisomy $8(+8)$ constituted the most common subset $(75 / 298,25.2 \%)$. The incidence of abnormal karyotypes was significantly higher in patients who were $\geq 51$ years old compared with those $<51$ years old, (54.8 vs. $34.7 \%$, respectively; $\mathrm{P}<0.05$ ). Based on World Health Organization (WHO) classification-based Prognostic Scoring System (WPSS) criteria, the incidence of poor-prognosis karyotypes was significantly higher $(17.4$ vs. $5.4 \%$; $\mathrm{P}<0.05)$ in the older patient group, and based on the Revised International Prognostic Scoring System (IPSS-R) criteria, the incidence of poor-/very poor-prognosis karyotypes was also significantly higher (17.4 vs. $6.6 \%$; $\mathrm{P}<0.05)$ in patients $\geq 51$ years old compared with younger ones. Based on the WHO classification of MDS subtypes, the incidence of abnormal karyotypes in patients with high percentages of bone marrow (BM) blasts [excess blasts (EB)-I + EB-II, $\geq 5 \%$ blasts] was significantly higher than that in patients with low percentages of BM blasts (those with single lineage dysplasia + multilineage dysplasia, $<5 \%$ blasts) ( 62.5 vs. $36.0 \%$; $\mathrm{P}<0.05)$. The incidence of poor-prognosis karyotypes based on WPSS criteria was
\end{abstract}

Correspondence to: Professor Xiaomei $\mathrm{Hu}$, Department of Hematology, Xiyuan Hospital, China Academy of Chinese Medical Sciences, 1 Xiyuancaochang, Haidian, Beijing 100091, P.R. China E-mail: huxiaomei_2@163.com

${ }^{*}$ Contributed equally

Key words: myelodysplastic syndrome, chromosome karyotype, cytogenetics, WHO classification, prognostic scoring system significantly higher in patients with high percentages of BM blasts than those with low percentages (22.0 vs. $6.9 \%$, respectively; $\mathrm{P}<0.05)$, and the incidence of poor-/very poor-prognosis karyotypes based on IPSS-R criteria was also significantly higher (23.0 vs. 7.4\%, respectively; $\mathrm{P}<0.05)$. These results demonstrate that +8 is the most common abnormal karyotype in Chinese patients with MDS. Age and the percentage of BM blasts are associated with the incidence of both abnormal karyotypes and karyotypes with poor prognosis. The results of cytogenetic abnormalities in this study will supplement the data on patients of MDS in China.

\section{Introduction}

Myelodysplastic syndrome (MDS) is a malignant clonal disease with high heterogeneity, which originates from hematopoietic stem cells. MDS is characterized by decreased numbers of peripheral blood cells and ineffective hematopoiesis, with a high risk of transformation to acute myeloid leukemia (AML) $(1,2)$. In view of the high heterogeneity of MDS, the World Health Organization (WHO) divides MDS into several subtypes (3): MDS with multilineage dysplasia (MDS-MLD), MDS with single lineage dysplasia (MDS-SLD), MDS with excess blasts-I (MDS-EB-I), MDS with excess blasts-II (MDS-EB-II), MDS with ring sideroblasts (MDS-RS), MDS, unclassifiable (MDS-U), and MDS with isolated del(5q). In addition to differences in morphology and clinical features, there are some differences in chromosome karyotype and prognosis among the different WHO subtypes (4). The annual incidence of MDS is $\sim 4 / 100,000$ population, but increases to $40-50 / 100,000$ for individuals aged $\geq 70$ years, which suggests that the incidence rate of MDS increases substantially with age $(5,6)$. The association of MDS with increasing age may be associated with genetic damage (7). Chromosome karyotype, an independent prognostic factor for MDS, has important significance in the diagnosis, treatment and prognosis of MDS management (8). In Asian, European and American countries, the incidence of MDS chromosomal abnormalities is $40-60 \%$ (9-12). However, the distribution of specific chromosomal abnormalities in MDS varies among different geographic regions (11-13). 
To better understand the biology of MDS in the Chinese population, the karyotypes of 665 Chinese patients with MDS were analyzed in the present study, and the different karyotype characteristics in different classification/prognosis groups were evaluated according to the WHO classification, WHO classification-based Prognostic Scoring System (WPSS) and Revised International Prognostic Scoring System (IPSS-R).

\section{Materials and methods}

Patients. A total of 665 patients with MDS who visited the Department of Hematology, Xiyuan Hospital, China Academy of Chinese Medical Sciences (Beijing, China) from January 2011 to September 2019 were included in the study. Criteria for inclusion in the study were as follows: A confirmed diagnosis of MDS; both bone marrow and peripheral blood blasts $\leq 19 \%$; and having not been treated. Patients with secondary MDS were excluded. Of the 665 patients, 355 were male and 310 were female, corresponding to a male-to-female ratio of 1.15 . The median age of the patients was 51 years (range, 6-86 years). According to the WHO classification, 423 patients (63.6\%) were classified as MDS-MLD, 120 patients $(18.0 \%)$ as MDS-EB-I, 80 patients $(12.0 \%)$ as MDS-EB-II, 24 patients (3.8\%) as MDS-SLD, 14 patients $(2.0 \%)$ as MDS-RS, 2 patients $(0.3 \%)$ as MDS-U and 2 patients $(0.3 \%)$ as MDS with isolated del $(5 q)$ (Table I). The study protocol was approved by the Clinical Research Ethics Committee of Xiyuan Hospital, China Academy of Chinese Medical Sciences. All patients provided written informed consent to participate in the study.

Diagnostic and classification criteria. All patients with MDS were diagnosed on the basis of the 2007 Vienna criteria (1), and their disease subtypes were classified according to $2016 \mathrm{WHO}$ criteria (3).

Cytogenetic prognostic groups. Cytogenetic prognostic groups were established based on the WPSS (2011) (14) and IPSS-R (2012) criteria (15). WPSS cytogenetic categories are as follows: Good-prognosis karyotype group, including normal, -Y, del(5q) and $\operatorname{del}(20 q)$; intermediate-prognosis karyotype group, including other abnormalities with the exception of those with good-prognosis or poor-prognosis karyotypes; and poor-prognosis karyotype group, including complex ( $\geq 3$ abnormalities) or chromosome 7 abnormalities. IPSS-R cytogenetic categories are as follows: Very good-prognosis karyotype group, including-Y and del(11q); good-prognosis karyotype group, including normal, del(5q), del(12p), del(20q), and $\operatorname{del}(5 \mathrm{q})$ with one additional abnormality; intermediate-prognosis karyotype group, including $\operatorname{del}(7 \mathrm{q})$, trisomy $8(+8),+19, \mathrm{i}(17 \mathrm{q})$ and any other single or double independent clones; poor-prognosis karyotype group, including-7, inv(3)/ $\mathrm{t}(3 \mathrm{q}) / \operatorname{del}(3 \mathrm{q}),-7 / \operatorname{del}(7 \mathrm{q})$ with one additional abnormality and complex abnormalities (3 abnormalities); very poor-prognosis karyotype group, including complex abnormalities ( $>3$ abnormalities).

Cytogenetic analysis. Fresh bone marrow (BM) cells were cultured in RPMI-1640 (HyClone; Cytiva) supplemented with $20 \%$ fetal calf serum (HyClone; Cytiva) and $3 \mu \mathrm{g} / \mathrm{ml}$ recombinant human granulocyte colony-stimulating factor (Xiamen Amoytop Biotech Co., Ltd.) at $37^{\circ} \mathrm{C}$ for $24 \mathrm{~h}$, and G-banding (16) was used for cytogenetic analysis. Karyotypes were classified and recorded according to the International System for Human Cytogenetic Nomenclature (2009) (17). At least 20 metaphases were analyzed in each sample, and the karyotypes with more than two chromosomal abnormalities were defined as complex karyotypes.

Statistical analysis. Data reported in the study relate to the time of diagnosis. All statistical analyses were performed using SPSS 23.0 (IBM Corp.) software. Independent-samples t-test was used to compare the means of two groups. $\chi^{2}$ tests or the Fisher's exact tests were used to compare proportions of two or multiple groups. The Bonferroni correction was used for multiple comparisons. $\mathrm{P}<0.05$ was considered to indicate a statistically significant difference. When the Bonferroni correction was used, the $\alpha$-level and P-values were adjusted.

\section{Results}

Chromosomal abnormalities and common abnormal karyotypes in patients with MDS. Among the 665 patients, 367 (55.2\%) had a normal karyotype while 298 (44.8\%) had abnormal karyotypes. The patients with abnormal karyotypes comprised 124 cases $(124 / 665,18.6 \%)$ with numerical abnormalities, 105 cases $(105 / 665,15.8 \%)$ with structural abnormalities, 55 cases $(55 / 665,8.3 \%)$ with complex abnormalities and 14 cases $(14 / 665,2.1 \%)$ with abnormalities both in number and structure (Table I, Fig. 1A).

Among the patients with single chromosome abnormalities, there were 75 cases $(75 / 665,11.3 \%)$ with $+8,38$ cases $(38 / 665,5.7 \%)$ with $\operatorname{del}(20 \mathrm{q}), 27$ cases $(27 / 665,4.1 \%)$ with $-7 / \operatorname{del}(7 q), 21$ cases $(21 / 665,3.2 \%)$ with $\operatorname{del}(5 q), 15$ cases $(15 / 665,2.3 \%)$ with $-Y, 8$ cases $(8 / 665,1.2 \%)$ with del(11q), 8 cases $(8 / 665,1.2 \%)$ with $+21,5$ cases $(5 / 665,0.8 \%)$ with $\operatorname{inv}(3) / \operatorname{del}(3 q)$ and 3 cases $(3 / 665,0.5 \%)$ with +19 .

Overall, +8 was the most frequent single abnormal karyotype in the present cohort. Of the 75 patients with +8 , $59(59 / 665,8.9 \%)$ had only the +8 abnormal karyotype, $11(11 / 665,1.7 \%)$ had +8 with one additional abnormality, and $5(5 / 665,0.8 \%)$ had +8 with complex abnormalities. Among the 27 patients with chromosome 7 abnormality, $8(8 / 665,1.2 \%)$ had isolated-7, $3(3 / 665,0.5 \%)$ had isolated del(7q), 10 (10/665, $1.5 \%)$ had-7 or $\operatorname{del}(7 q)$ with one additional abnormality, and $6(6 / 665,0.9 \%)$ had-7 or del(7q) with complex abnormalities. Among the 21 patients with $\operatorname{del}(5 q), 12(12 / 665,1.8 \%)$ had $\operatorname{del}(5 q)$ in isolation, $4(4 / 665,0.6 \%)$ had $\operatorname{del}(5 q)$ with one additional abnormality, and $5(5 / 665,0.8 \%)$ had $\operatorname{del}(5 q)$ with complex abnormalities (Fig. 1B). Among the 55 patients with complex karyotypes, $26(26 / 665,3.9 \%)$ had 3 chromosomal abnormalities and $29(29 / 665,4.4 \%)$ had $>3$ abnormalities.

Cytogenetic risk based on two MDS prognostic scoring systems. When the prognoses of the patients were evaluated on the basis of their karyotype analysis using WPSS criteria, $416(416 / 665,62.6 \%)$ of all patients were classified as having a good prognosis, $173(173 / 665,26.0 \%)$ as having intermediate prognosis and $76(76 / 665,11.4 \%)$ as having a poor prognosis 
Table II. Comparison of median age and PB blasts between normal and abnormal karyotype groups.

\begin{tabular}{lcc}
\hline Group & Age, years & PB blasts, \% \\
\hline Normal karyotype & $45.03 \pm 19.19$ & $3.26 \pm 4.34$ \\
Abnormal karyotypes & $53.54 \pm 17.94$ & $3.99 \pm 4.59$ \\
P-value & $<0.001$ & 0.395
\end{tabular}

Data are expressed as mean \pm standard deviation. PB, peripheral blood.

(Fig. 2A). The patient cohort was also evaluated using the IPSS-R system, which classified $12(12 / 665,1.8 \%)$ patients as having very good cytogenetic prognosis, 407 (407/665, 61.2\%) as having a good prognosis, $166(166 / 665,25.0 \%)$ as having an intermediate prognosis, $51(51 / 665,7.6 \%)$ as having a poor prognosis and $29(29 / 665,4.4 \%)$ as having a very poor prognosis (Fig. 2B).

Incidence of chromosomal abnormalities in different age groups. Chromosomal abnormalities were examined in patients with MDS of different age groups, and differences were observed in the incidence of abnormal karyotypes across the age groups. The incidence of abnormal karyotypes was $28 \%(14 / 50)$ in $\leq 20$-year-old patients, $25.8 \%$ (24/93) in patients $21-30$ years old, $36.3 \%$ (33/91) in patients $31-40$ years old, $45.4 \%$ (44/97) in patients $41-50$ years old, $50.4 \%(62 / 123)$ in patients 51-60 years old, 57.5\% (69/120) in patients 61-70 years old, $58.0 \%$ (40/69) in patients $71-80$ years old, and $54.5 \%(12 / 22)$ in $>80$-year-old patients (Fig. 3A).

All patients were divided into two age groups using the median age of 51 years as the cutoff. Among the 331 patients aged $<51$ years, $115(115 / 331,34.7 \%)$ had abnormal karyotypes, $18(18 / 331,5.4 \%)$ had poor-prognosis karyotypes based on WPSS criteria and $22(22 / 331,6.6 \%)$ had poor-/very poor-prognosis karyotypes based on IPSS-R criteria. Among the 334 patients who were $\geq 51$ years old, $183(183 / 334,54.8 \%)$ had abnormal karyotypes, 58 (58/334, 17.4\%) had poor-prognosis karyotypes based on WPSS criteria and 58 (58/334, 17.4\%) had poor-/very poor-prognosis karyotypes based on IPSS-R criteria. The incidence of abnormal karyotypes was significantly higher in patients aged $\geq 51$ years compared with those aged $<51$ years ( 54.8 vs. $34.7 \%$, respectively; $\mathrm{P}<0.05)$, and the incidence of poor-prognosis karyotypes based on WPSS criteria (17.4 vs. 5.4\%, respectively) and of poor-/very poor-prognosis karyotypes based on IPSS-R criteria (17.4 vs. 6.6\%, respectively) were also significantly higher $(\mathrm{P}<0.05)$ in patients aged $\geq 51$ years than in younger patients (Fig. 3B-D).

The mean ages of patients with normal and abnormal karyotypes were also compared, and the results revealed that the mean age of patients with abnormal karyotypes was significantly higher compared with that of patients with a normal karyotype $(53.54 \pm 17.94$ vs. $45.03 \pm 19.19$ years, respectively; $\mathrm{P}<0.001$; Table II).

Incidence of chromosomal abnormalities across different WHO classification groups. When classified using the 

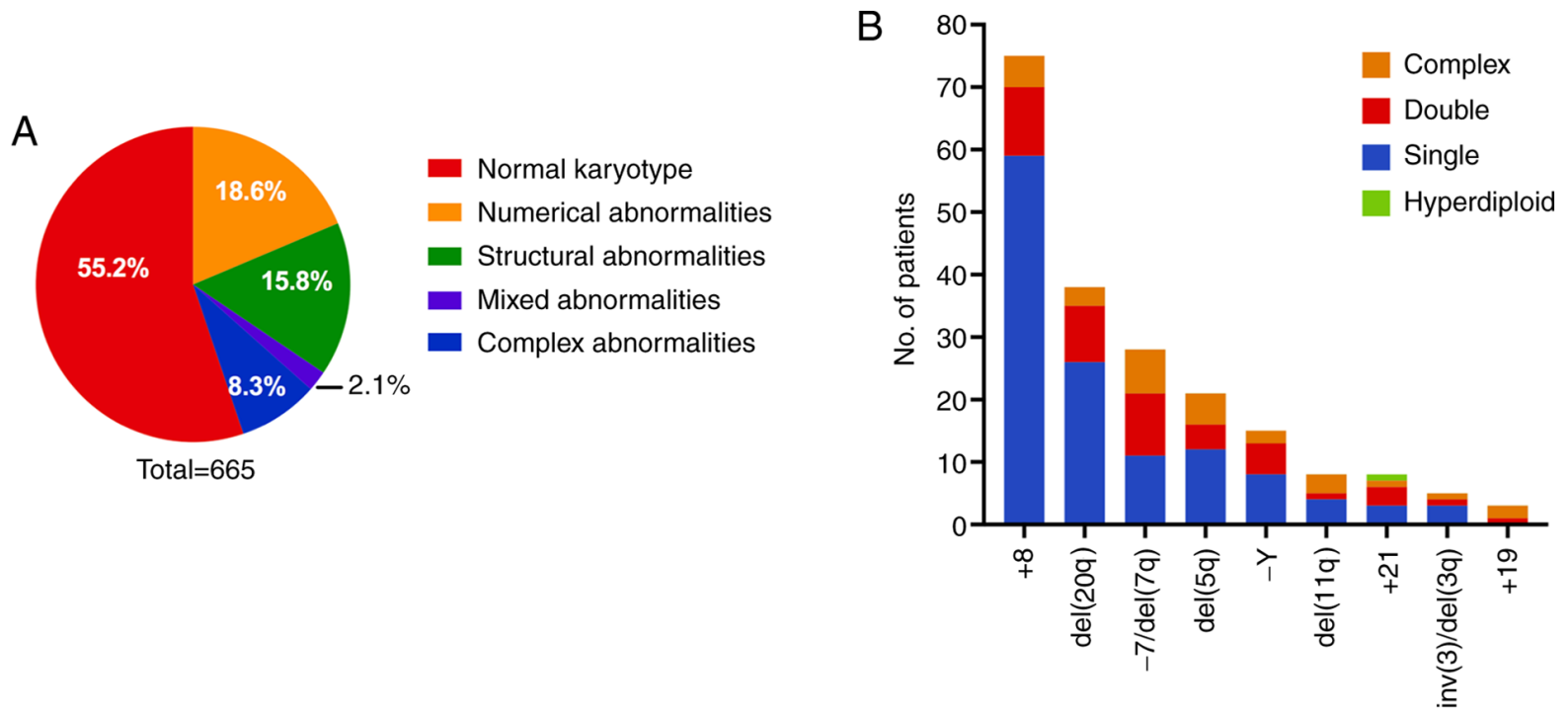

Figure 1. Abnormal karyotypes in patients with myelodysplastic syndrome. (A) Percentages of different types of chromosomal abnormality. (B) Numbers of patients with various single chromosome abnormalities. Mixed abnormalities refers to numerical and structural abnormalities.

A

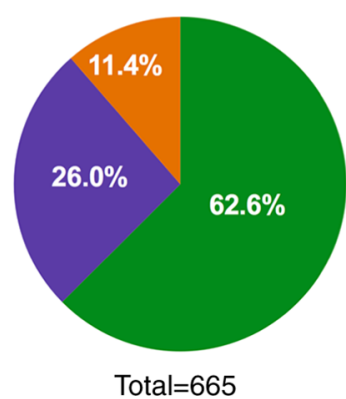

B

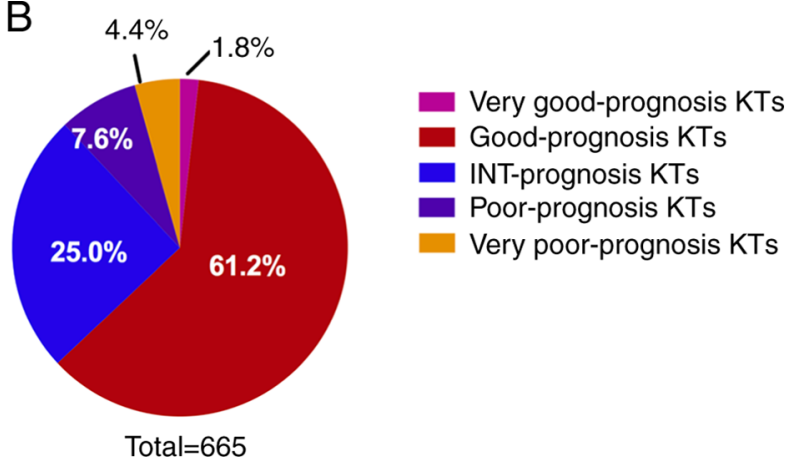

Figure 2. Incidence of chromosomal abnormalities based on prognostic scoring systems for myelodysplastic syndrome. Incidence based on (A) World Health Organization classification-based Prognostic Scoring System criteria and (B) Revised International Prognostic Scoring System criteria. KTs, karyotypes; INT, intermediate.

WHO (2016) criteria, the cohort of 665 patients with MDS was segregated into the following groups: SLD, 24 cases including 8 cases $(8 / 24,33.3 \%)$ with abnormal karyotypes; MLD, 423 cases including 153 cases $(153 / 423,36.2 \%)$ with abnormal karyotypes; RS, 14 cases including 9 cases $(9 / 14,64.3 \%)$ with abnormal karyotypes; EB-I, 120 cases including 78 cases $(78 / 120,65.0 \%)$ with abnormal karyotypes; EB-II, 80 cases including 47 cases $(47 / 80,58.7 \%)$ with abnormal karyotypes; MDS with isolated $\operatorname{del}(5 q), 2$ cases including 1 case with isolated $\operatorname{del}(5 q)$ and 1 case with one additional abnormality; and MDS-U, 2 cases including 1 case $(1 / 2,50 \%)$ with one abnormality.

As shown in Table III, the +8 abnormality was found in all WHO subtypes with the exception of MDS-U and MDS with isolated $\operatorname{del}(5 q)$, and was more prevalent than other abnormalities in those subtypes; del(20q) was found in all subtypes with the exception of MDS-U; and -7/del(7q) and $\operatorname{del}(5 q)$ were more common in EB-I and EB-II than in other subtypes.

The incidence of abnormal karyotypes in cases of EB-I (65.0\%) and EB-II (58.7\%) was significantly higher compared with that in MLD (36.2\%) (adjusted $\mathrm{P}<0.005)$. Furthermore, the incidence of abnormal karyotypes in EB-I (65.0\%) was significantly increased compared with that in SLD (33.3\%) (adjusted $\mathrm{P}<0.005)$. However, no significant differences were identified in the incidence of abnormal karyotypes among the other subtypes of MDS (Fig. 4A).

The incidence of abnormal karyotypes in patients with high percentages of BM blasts (EB-I + EB-II, $\geq 5 \%$ blasts) was significantly higher compared with that in patients with low percentages of BM blasts (SLD + MLD, $<5 \%$ blasts), at 62.5 and $36.0 \%$, respectively $(\mathrm{P}<0.05)$. The incidence of poor-prognosis karyotypes based on WPSS criteria was significantly higher in patients with high percentages of BM blasts than in those with low BM blast percentages (22.0 vs. $6.9 \%$, respectively; $\mathrm{P}<0.05$ ), as was the incidence of poor-/very poor-prognosis karyotypes based on IPSS-R criteria (23.0 vs. $7.4 \%$, respectively; $\mathrm{P}<0.05$ ) (Fig. 4B-D).

In addition to BM blasts, the mean percentages of peripheral blood blasts were also compared between patients with normal and abnormal karyotypes, and no significant difference was identified (Table II). 
Table III. Frequency of common chromosomal abnormalities among WHO subtypes of MDS.

\begin{tabular}{|c|c|c|c|c|c|c|c|}
\hline \multirow{3}{*}{$\begin{array}{l}\text { Subtypes } \\
\text { MLD }\end{array}$} & \multirow{3}{*}{$\begin{array}{c}\text { No. of patients } \\
423\end{array}$} & \multicolumn{6}{|c|}{ No. of frequent chromosomal abnormalities (\%) } \\
\hline & & \multirow{2}{*}{$\begin{array}{l}\text { Abnormal } \\
153(36.2)\end{array}$} & \multirow{2}{*}{$\begin{array}{c}+8 \\
39 \quad(9.2)\end{array}$} & \multirow{2}{*}{$\frac{\operatorname{del}(20 q)}{24(5.7)}$} & \multirow{2}{*}{$\frac{-7 / \operatorname{del}(7 q)}{15(3.5)}$} & \multicolumn{2}{|c|}{$\operatorname{del}(5 q)$} \\
\hline & & & & & & 8 & (1.9) \\
\hline SLD & 24 & $8(33.3)$ & 1 (4.2) & $1(4.2)$ & 0 & 0 & \\
\hline EB-I & 120 & $78(65.0)$ & $20(16.7)$ & $6(5.0)$ & $6 \quad(5.0)$ & 5 & $(4.2)$ \\
\hline EB-II & 80 & $47(58.7)$ & $11(13.8)$ & $3(3.8)$ & $5 \quad(6.3)$ & 5 & (6.3) \\
\hline MDS-RS & 14 & $9(64.3)$ & 4 (28.6) & $3(21.4)$ & 0 & 1 & (7.1) \\
\hline MDS-U & 2 & $1(50.0)$ & 0 & 0 & $1(50.0)$ & 0 & \\
\hline $\operatorname{MDS} \operatorname{del}(5 q)$ & 2 & $2(100.0)$ & 0 & $1(50.0)$ & 0 & & 100.0) \\
\hline
\end{tabular}

MDS, myelodysplastic syndrome; WHO, World Health Organization; MLD, multilineage dysplasia; SLD, single lineage dysplasia; EB-I, excess blasts-I; EB-II, excess blasts-II; MDS-RS, MDS with ring sideroblasts; MDS-U, MDS, unclassifiable; MDS del(5q), MDS with isolated $\operatorname{del}(5 q)$.
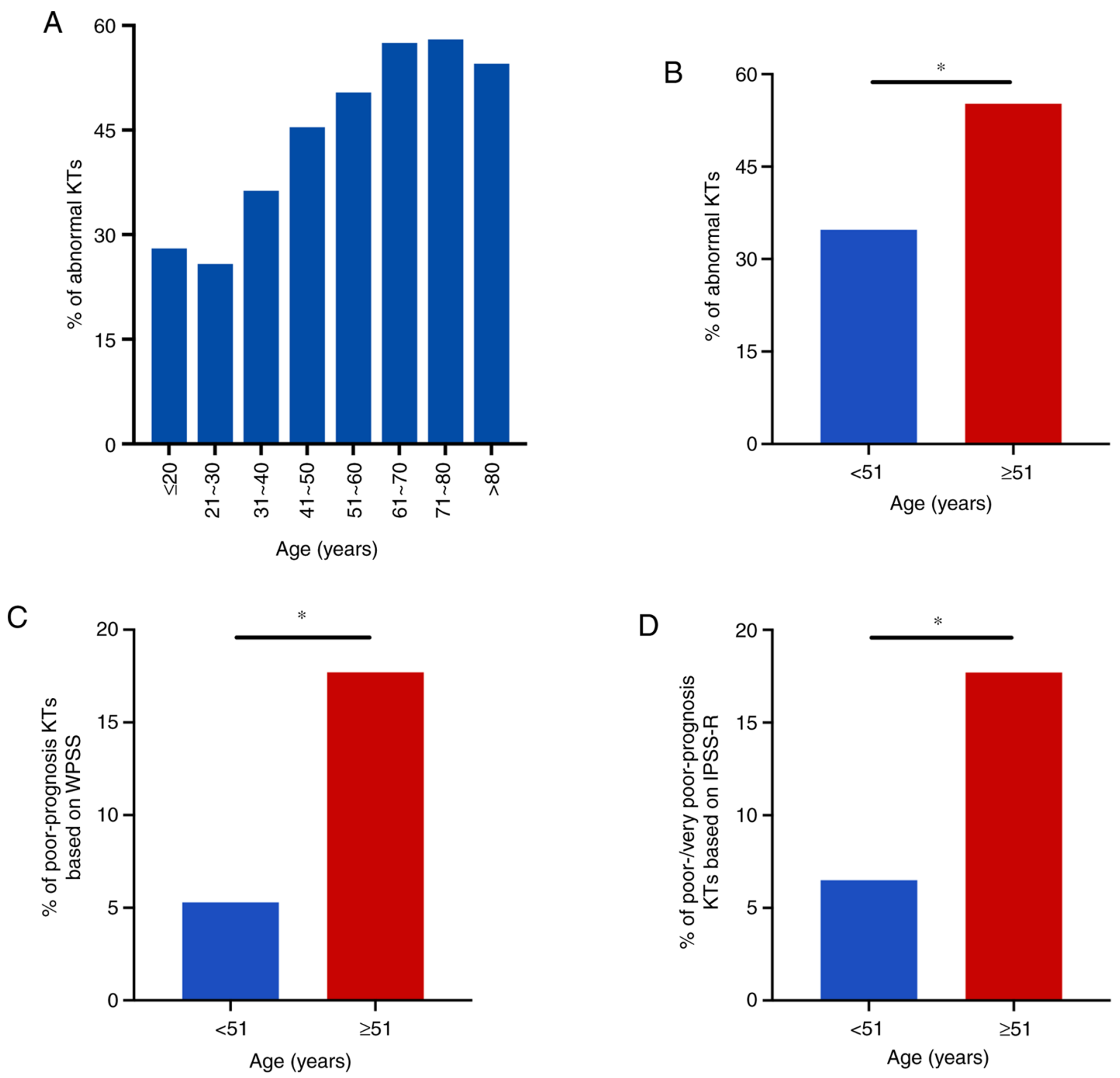

Figure 3. Incidence of chromosomal abnormalities in different age groups. (A) Incidence of abnormal KTs in different age groups. Incidence of (B) abnormal KTs, (C) poor-prognosis KTs according to WPSS criteria and (D) poor- and very poor-prognosis KTs according to IPSS-R criteria in patients divided into two groups according to median age. * $\mathrm{P}<0.05$. KTs, karyotypes; WPSS, WHO classification-based Prognostic Scoring System; IPSS-R, Revised International Prognostic Scoring System. 
A

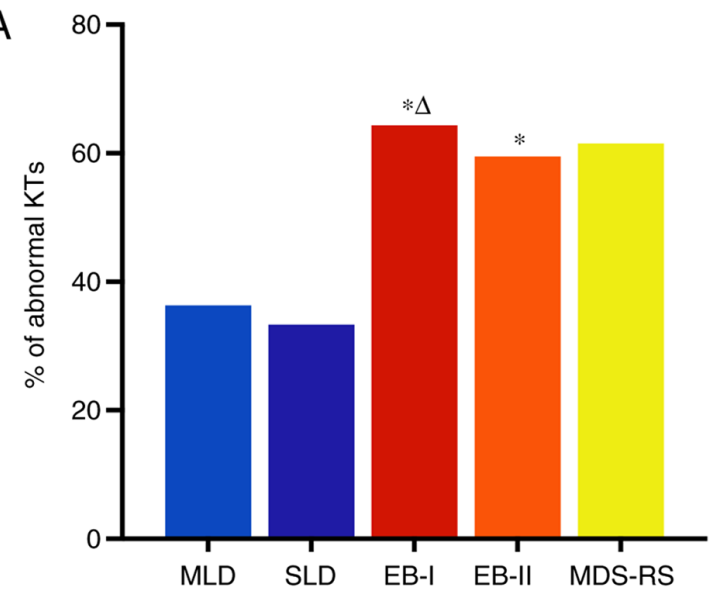

C

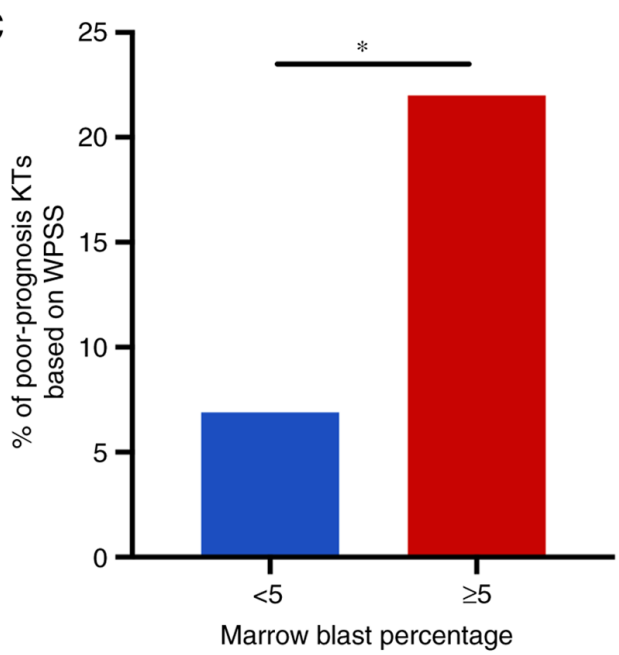

$\mathrm{B}$
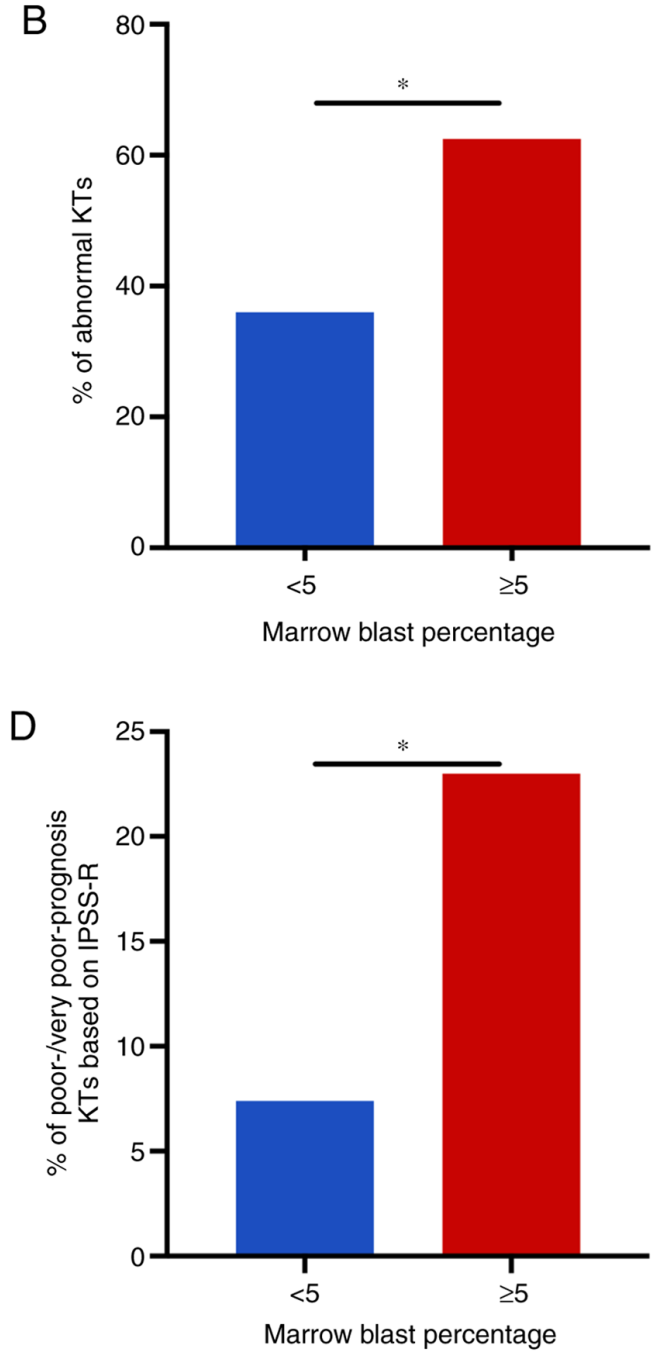

Figure 4. Incidence of chromosomal abnormalities across different WHO classification groups. (A) Comparison of different WHO subtypes of myelodysplastic syndrome. $\mathrm{P}<0.001$ among all WHO subtypes (4 degrees of freedom). Pairwise comparisons of different WHO subtypes: MLD vs. SLD, P=0.778; MLD vs. EB-I, P<0.001; MLD vs. EB-II, P<0.001; MLD vs. MDS-RS, P=0.032; SLD vs. EB-I, P=0.004; SLD vs. EB-II, P=0.029; SLD vs. MDS-RS, P=0.094; EB-I vs. EB-II, $\mathrm{P}=0.371$; EB-I vs. MDS-RS, $\mathrm{P}=1.000$; EB-II vs. MDS-RS, $\mathrm{P}=0.697$. After Bonferroni correction, the adjusted $\mathrm{P}<0.005$ was used to indicate a statistically significant difference among the multiple pairwise comparisons of different WHO subtypes. ${ }^{*} \mathrm{P}<0.005$ vs. MLD, ${ }^{\triangle} \mathrm{P}<0.005$ vs. SLD. (B) Incidence of (B) abnormal KTs, (C) poor-prognosis KTs according to WPSS criteria and (D) poor- and very poor-prognosis KTs according to IPSS-R criteria in patients divided into two groups according to the percentage of bone marrow blasts. " $\mathrm{P}<0.05$. WHO, World Health Organization; MLD, multilineage dysplasia; SLD, single lineage dysplasia; EB-I, excess blasts-I; EB-II, excess blasts-II; MDS-RS, MDS with ring sideroblasts; KTs, karyotypes; WPSS, WHO classification-based Prognostic Scoring System; IPSS-R, Revised International Prognostic Scoring System.

\section{Discussion}

The median age of MDS onset differs between Asian and Western countries. MDS is considered to be a disease associated with aging in Western countries, and the median and mean ages at diagnosis in Western countries are $\sim 70$ years $(7,18,19)$. However, the median age at which patients are diagnosed with MDS in Asian countries is lower, and ranges from 49 to 58 years in China $(11,20)$. In the present study, the median age was 51 years, which is consistent with previous studies.

According to the literature, $40-60 \%$ of patients with MDS possess cytogenetic abnormalities in both Chinese and Western populations (10-12). The results of the present study indicate that the incidence of chromosomal abnormalities in Chinese patients with MDS was $44.8 \%$, corroborating previous data.

Previous studies have shown that the most common karyotype abnormalities differ between patients with MDS in
China and those in Western countries. While +8 is the most common karyotype abnormality in patients with MDS from China and other Asian countries, the most common karyotype abnormality in Western patients with MDS is del(5q) (21-23). In the present study, 75 patients had the +8 abnormality, accounting for $25.2 \%$ of the abnormal karyotypes. This genetic difference may partially explain the differences in clinical characteristics and prognosis between Asian and Western patients with MDS, and suggests that targeted treatments should be considered for different patient groups with different karyotype abnormalities.

There are other differences in the distribution of prognostic karyotypes between Chinese and Western patients with MDS. In data from Western populations (15), the IPSS-R cytogenetic categories were found to be distributed as follows: $4 \%$ of patients had very good-prognosis karyotypes; $72 \%$ of patients had good-prognosis karyotypes; $13 \%$ of patients 
had intermediate-prognosis karyotypes; $4 \%$ of patients had poor-prognosis karyotypes; and $7 \%$ of patients had very poor-prognosis karyotypes. By comparison, Qu et al (24) reported that in Chinese populations, the distributions of the IPSS-R cytogenetic categories were as follows: $2 \%$ of patients had very good-prognosis karyotypes; $43 \%$ had good-prognosis karyotypes; $36 \%$ had intermediate-prognosis karyotypes; $7 \%$ had poor-prognosis karyotypes; and $12 \%$ had very poor-prognosis karyotypes. The data from the present study revealed that very good-prognosis karyotypes were present in $1.8 \%$ of patients, good-prognosis karyotypes in $61.2 \%$, intermediate-prognosis karyotypes in $25.0 \%$, poor-prognosis karyotypes in $7.6 \%$ and very poor-prognosis karyotypes in $4.4 \%$. The differences in the data between Chinese and Western patients with MDS are mainly in the karyotypes with good and intermediate prognosis, which may be because $\operatorname{del}(5 q)$ is the most common abnormality in the West while +8 is the most common abnormality in Chinese patients. However, the data in the present study differ from the results reported by Qu et al (24), suggesting that further study using larger patient cohorts is warranted.

Previous studies have shown that aging is an adverse prognostic factor for MDS $(25,26)$. The present study identified that the mean age of patients with abnormal karyotypes was higher than that of patients with a normal karyotype. Furthermore, the incidence rates of chromosomal abnormalities and karyotypes with poor prognosis were both significantly increased in $\geq 51$-year-old patients. Thus, it may be inferred that both chromosomal abnormalities and karyotypes with poor prognosis are more common in Chinese patients with MDS who are $\geq 51$ years old than in younger patients. Therefore, it appears that the adverse effects of aging on prognosis may be associated with the higher incidence of poor-prognosis karyotypes in older patients.

As the disease progresses, the incidence of chromosomal abnormalities in patients with MDS gradually increases from $30-40 \%$ in the early stage of the disease to $50-70 \%$ in the advanced stage $(12,20)$. The progression of MDS involves an increase in the number of blasts and a reduction in the number of peripheral blood cells, which can eventually develop into AML or BM failure. A previous study has proposed that the incidence of abnormal karyotypes in patients with early MDS is lower than that in patients with advanced MDS, and secondary chromosomal abnormalities are likely to occur in advanced cases (27). Chromosomal abnormalities can also affect the development of AML in patients with MDS $(15,27)$. According to the WHO classification, the BM blast percentages in the SLD and MLD subtypes of MDS are $<5 \%$, while in the EB-I and EB-II subtypes they are $\geq 5 \%$. The data in the present study showed clear differences in the incidence of chromosomal abnormalities and of poor-prognosis karyotypes between the subtypes with $<5 \%$ BM blasts and those with $\geq 5 \%$ blasts. Specifically, chromosomal abnormalities and poor-prognosis karyotypes were more common in the patients with a high percentage of blasts, which is consistent with previous reports $(23,24)$.

The evolution of chromosome karyotype is closely associated with the progression of MDS, which may be related to the genetic instability caused by chromosomal aberrations $(7,28)$. As a result of genomic alteration and clonal expansion, some malignant clones may gain proliferation advantages and be prone to transformation into leukemic clones, while other clones may be eliminated by selection mechanisms $(7,29)$.

While chromosome karyotype is important, it is just one of several independent factors affecting the prognosis of MDS. It should be considered together with other prognostic factors, including the degree of cytopenia in peripheral blood, the percentage of BM blasts and WHO subtypes. It is clear that the incidence and types of chromosomal abnormalities vary among different regions of the world. Some countries have proposed a more suitable prognosis scoring system for their own populations $(30,31)$. However, at present, there is no MDS prognosis score system suitable for Chinese populations. The present study data, together with larger datasets collected in future studies, will collectively contribute to the formulation of a more accurate MDS prognosis scoring system for Chinese patients in the future.

\section{Acknowledgements}

The authors would like to thank Professor Xiaosheng Wu (Department of Biochemistry and Molecular Biology, Mayo Clinic, Rochester, MN, USA) for English language editing.

\section{Funding}

This study was supported by grants from the National Natural Science Foundation of China (grant nos. 81673821 and 81774142) and the Special Research Foundation of Central Level Public Scientific Research Institutes (grant no. ZZ10-016).

\section{Availability of data and materials}

All data and materials analyzed during the current study are included in this published article.

\section{Authors' contributions}

$\mathrm{XH}$ designed the study; XW, WL, MW and XG analyzed the data and wrote the manuscript; XW, XG and XY performed the cytogenetic analyses; $\mathrm{XH}$ gave the final karyotype approval; WL, TF, YML, HX, HW, SZ, RQ, CL, XT, YL, ZC, LL, YX and RM collected the clinical data, analyzed and interpreted the data; HW, YX and RM performed the morphologic review and gave the final approval. XH checked the final manuscript. All authors read and approved the final manuscript.

\section{Ethics approval and consent to participate}

The study protocol was approved by the Clinical Research Ethics Committee of Xiyuan Hospital, China Academy of Chinese Medical Sciences. All patients provided written informed consent to participate in the study.

\section{Patient consent for publication}

Not applicable.

\section{Competing interests}

The authors declare that they have no competing interests. 


\section{References}

1. Vanlet P, Horny HP, Bennet JM, Fonatsch C, Germing U, Greenberg P, Haferlach T, Haase D, Kolb HJ, Krieger O, et al: Definitions and standards in the diagnosis and treatment of myelodysplastic syndromes: Consensus statements and report from a working conference. Leuk Res 31: 727-736, 2007.

2. AdèS L, Itzykson R and Fenaux P: Myelodysplastic syndromes. Lancet 383: 2239-2252, 2014.

3. Arber DA, Orazi A, Hasserjian R, Thiele J, Borowitz MJ, Le Beau MM, Bloomfield CD, Cazzola M and Vardiman JW: The 2016 revision to the World Health Organization classification of myeloid neoplasms and acute leukemia. Blood 127: 2391-2405, 2016.

4. Malcovati L, Germing U, Kuendgen A, Della Porta MG, Pascutto C, Invernizzi R, Giagounidis A, Hildebrandt B, Bernasconi P, Knipp S, et al: Time-dependent prognostic scoring system for predicting survival and leukemic evolution in myelodysplastic syndromes. J Clin Oncol 25: 3503-3510, 2007.

5. Neukirchen J, Schoonen WM, Strupp C, Gattermann N, Aul C, Haas R and Germing U: Incidence and prevalence of myelodysplastic syndromes: Data from the Düsseldorf MDS-registry. Leuk Res 35: 1591-1596, 2011.

6. Zeidan AM, Shallis RM, Wang R, Davidoff A and Ma XM: Epidemiology of myelodysplastic syndromes: Why characterizing the beast is a prerequisite to taming it. Blood Rev 34: 1-15, 2019.

7. Corey SJ, Minden MD, Barber DL, Kantarjian H, Wang JC and Schimmer AD: Myelodysplastic syndromes: The complexity of stem-cell diseases. Nat Rev Cancer 7: 118-129, 2007.

8. Schanz J, Tüchler H, Solé F, Mallo M, Luño E, Cervera J, Granada I, Hildebrandt B, Slovak ML, Ohyashiki K, et al: New comprehensive cytogenetic scoring system for primary myelodysplastic syndromes (MDS) and oligoblastic acute myeloid leukemia after MDS derived from an international database merge. J Clin Oncol 30: 820-829, 2012.

9. Bernasconi P, Klersy C, Boni M, Cavigliano PM, Calatroni S, Giardini I, Cavigliano PM, Calatroni S, Giardini I, Rocca B, et al: World Health Organization classification in combination with cytogenetic markers improves the prognostic stratification of patients with de novo primary myelodysplastic syndromes. Br J Haematol 137:193-205, 2007.

10. Xiao Y, Wei J, Chen Y, Zhang KJ, Zhou JF and Zhang YC: Trisomy 8 is the most frequent cytogenetic abnormality in de novo myelodysplastic syndrome in China. Onkologie 35: 100-106, 2012

11. Wang H, Wang XQ, Xu XP and Lin GW: Cytogenetic features and prognosis analysis in Chinese patients with myelodysplastic syndrome: A multicenter study. Ann Hematol 89: 535-544, 2010.

12. Sole F, Espinet B, Sanz GF, Cervera J, Calasanz MJ, Luño E, Prieto F, Granada I, Hernández JM, Cigudosa JC, et al: Incidence, characterization and prognostic significance of chromosomal abnormalities in 640 patients with primary myelodysplastic syndromes. Grupo Cooperativo Español de Citogenética Hematológica. Br J Haematol 108: 346-356, 2000.

13. Matsuda A, Germing U, Jinnai I, Misumi M, Kuendgen A, Knipp S, Aivado M, Iwanaga M, Miyazaki Y, Tsushima H, et al: Difference in clinical features between Japanese and German patients with refractory anemia in myelodysplastic syndromes. Blood 106: 2633-2640, 2005.

14. Malcovati L, Della Porta MG, Strupp C, Ambaglio I, Kuendgen A, Nachtkamp K, Travaglino E, Invernizzi R, Pascutto C, Lazzarino M, et al: Impact of the degree of anemia on the outcome of patients with myelodysplastic syndromes and its integration into the WHO classification-based Prognostic Scoring System (WPSS). Haematologica 96: 1433-1440, 2011.

15. Greenberg PL, Tuechler H, Schanz J, Sanz G, Garcia-Manero G, Solé F, Bennett JM, Bowen D, Fenaux P, Dreyfus F, et al: Revised international prognostic scoring system for myelodysplastic syndromes. Blood 120: 2454-2465, 2012.

16. Seabright M: A rapid banding technique for human chromosomes. Lancet 2: 971-972, 1971.

17. Shaffer LG, Slovak ML and Campbell LJ (eds.): ISCN: An International System for Human Cytogenetic Nomenclature. S. Karger, Basel, 2009.
18. Pozdnyakova O, Miron PM, Tang G, Walter O, Raza A, Woda B and Wang SA: Cytogenetic abnormalities in a series of 1029 patients with primary myelodysplastic syndromes: A report from the US with a focus on some undefined single chromosomal abnormalities. Cancer 113: 3331-3340, 2008.

19. Germing U, Gattermann N, Strupp C, Aivado M and Aul C: Validation of the WHO proposals for a new classification of primary myelodysplastic syndromes: A retrospective analysis of 1600 patients. Leuk Res 24: 983-992, 2000.

20. Chen B, Zhao WL, Jin J, Xue YQ, Cheng X, Chen XT, Cui J, Chen ZM, Cao Q, Yang G, et al: Clinical and cytogenetic features of 508 Chinese patients with myelodysplastic syndrome and comparison with those in Western countries. Leukemia 19: 767-775, 2005.

21. Wu L, Song L, Xu L, Chang C, Xu F, Wu D, He Q, Su J, Zhou L, Xiao C, et al: Genetic landscape of recurrent ASXL1, U2AF1, SF3B1, SRSF2, and EZH2 mutations in 304 Chinese patients with myelodysplastic syndromes. Tumour Biol 37: 4633-4640, 2016.

22. Lai YY, Huang XJ, Li J, Zou P, Xu ZF, Sun H, Shao ZH, Zhou DB, Chen FP, Liu ZG, et al: Standardized fluorescence in situ hybridization testing based on an appropriate panel of probes more effectively identifies common cytogenetic abnormalities in myelodysplastic syndromes than conventional cytogenetic analysis: A multicenter prospective study of 2302 patients in China. Leuk Res 39: 530-535, 2015.

23. Haase D, Germing U, Schanz J, Pfeilstöcker M, Nösslinger T, Hildebrandt B, Kundgen A, Lübbert M, Kunzmann R, Giagounidis AA, et al: New insights into the prognostic impact of the karyotype in MDS and correlation with subtypes: Evidence from a core dataset of 2124 patients. Blood 110: 4385-4395, 2007.

24. Qu S, Xu Z, Zhang Y, Qin T, Cui R and Xiao Z: Impacts of cytogenetic categories in the Revised International Prognostic Scoring System on the prognosis of primary myelodysplastic syndromes: Results of a single-center study. Leuk Lymphoma 53: 940-946, 2012.

25. Xu Y, Li Y, Xu Q, Chen Y, Lv N, Jing Y, Dou L, Bo J, Hou G, Guo J, et al: Implications of mutational spectrum in myelodysplastic syndromes based on targeted next-generation sequencing. Oncotarget 8: 82475-82490, 2017.

26. Gangat N, Mudireddy M, Lasho TL, Finke CM, Nicolosi M, Szuber N, Patnaik MM, Pardanani A, Hanson CA, Ketterling RP and Tefferi A: Mutations and prognosis in myelodysplastic syndromes: Karyotype-adjusted analysis of targeted sequencing in 300 consecutive cases and development of a genetic risk model. Am J Hematol 93: 691-697, 2018.

27. Bernasconi P, Klersy C, Boni M, Cavigliano PM, Giardini I, Rocca B, Zappatore R, Dambruoso I, Calvello C, Caresana M and Lazzarino M: Does cytogenetic evolution have any prognostic relevance in myelodysplastic syndromes? A study on 153 patients from a single institution. Ann Hematol 89: 545-551, 2010.

28. Willman CL: Molecular genetic features of myelodysplastic syndromes (MDS). Leukemia 12 (Suppl 1): S2-S6, 1998.

29. Greenberg PL: Apoptosis and its role in the myelodysplastic syndromes: Implications for disease natural history and treatment. Leuk Res 22: 1123-1136, 1998.

30. Solé F, Luño E, Sanzo C, Espinet B, Sanz GF, Cervera J, Calasanz MJ, Cigudosa JC, Millà F, Ribera JM, et al: Identification of novel cytogenetic markers with prognostic significance in a series of 968 patients with primary myelodysplastic syndromes. Haematologica 90: 1168-1178, 2005.

31. Kantarjian H, O'Brien S, Ravandi F, Cortes J, Shan J, Bennett JM, List A, Fenaux P, Sanz G, Issa JP, et al: Proposal for a new risk model in myelodysplastic syndrome that accounts for events not considered in the original International Prognostic Scoring System. Cancer 113: 1351-1361, 2008.

This work is licensed under a Creative Commons Attribution-NonCommercial-NoDerivatives 4.0 International (CC BY-NC-ND 4.0) License. 